"(C) 2015 IEEE. Personal use of this material is permitted. Permission from IEEE must be obtained for all other uses, in any current or future media, including reprinting/republishing this material for advertising or promotional purposes, creating new collective works, for resale or redistribution to servers or lists, or reuse of any copyrighted component of this work in other works." 


\title{
CogBoost: Boosting for Fast Cost-sensitive Graph Classification
}

\author{
Shirui Pan, Jia Wu, and Xingquan Zhu, Senior Member, IEEE
}

\begin{abstract}
Graph classification has drawn great interests in recent years due to the increasing number of applications involving objects with complex structural relationships. To date, all existing graph classification algorithms assume, explicitly or implicitly, that misclassifying instances in different classes incurs an equal amount of cost/risk, which is often not the case in real-life applications (where misclassifying a certain class of samples, such as diseased patients, is subject to more expensive costs than others). Although cost-sensitive learning has been extensively studied, all methods are based on data with instance-feature representation. Graphs, however, do not have features available for learning and the possible feature space of graph data are likely infinite and needs to be carefully explored in order to favor classes with a higher cost. Furthermore, current graph classification models are designed for small graph datasets and can hardly scale to the increase of the training set size. In this paper, we propose, CogBoost, a fast cost-sensitive graph classification algorithm, where the goal is to minimize the misclassification costs (instead of the errors) and to achieve fast learning speed for large scale graph datasets. To minimize the misclassification costs, CogBoost iteratively selects the most discriminative subgraph by considering costs of different classes, and then solves a linear programming problem in each iteration by using Bayes decision rule based optimal loss function. In addition, a cutting plane algorithm is derived to speed up the solving of linear programs for fast learning on large graph datasets. Experiments and comparisons on real-world large graph datasets demonstrate the effectiveness and the efficiency of our algorithm.
\end{abstract}

Index Terms-Graph classification, Cost-sensitive learning, Subgraphs, Boosting, Cutting plane algorithm, Large scale graphs

\section{INTRODUCTION}

$\mathrm{D}$ UE to the rapid advancement in the data collection technology, recent years have witnessed an increasing number of applications with complex structural relationships inside the data, e.g., chemical compounds [1], social networks [2], and scientific publications [3]. Different from traditional data which are represented in deterministic feature space by using an instance-feature representation, structural data are not represented by using attribute vectors, but by graphs with dependency relationships between objects. Because graphs do not have features immediately available for learning, this challenge has recently motivated a number of works using different designs for graph classification, which either learn some global similarities between graphs measured by graph kernels and graph embedding [1], [4], or select some informative subgraphs as features to differentiate graphs in different categories [5], [6], [7], [8], [9], [3], [10], [11]. However, all existing methods for graph classification may suffer two fundamental issues, i.e.,

S. Pan is with the College of Information Engineering, Northwest $A \& F$ University, Yangling, 712100, China, and also with the Centre for Quantum Computation \& Intelligent Systems, FEIT, University of Technology Sydney, Sydney, NSW 2007, Australia (e-mail: shirui.pan@student.uts.edu.au)

$J$. Wu is with the Department of Computer Science, China University of Geosciences, Wuhan 430074, P.R. China, and also with the Centre for Quantum Computation and Intelligent Systems, FEIT, University of Technology, Sydney, Sydney, NSW 2007, Australia (e-mail: jia.wu@student.uts.edu.au).

$X$. Zhu is with the Department of Computer and Electrical Engineering and Computer Science, Florida Atlantic University, Boca Raton, FL 33431,USA (e-mail: xzhu3@fau.edu).

Manuscript received $* * * *, 2014$; revised $* * * *$, accepted $* * 201-$. ineffective for cost-sensitive classification and inefficient for large graphs, as stated as follows:

\subsection{Cost-Sensitive Graph Classification}

For graph classification, all existing methods assume, explicitly or implicitly, that misclassifying a positive graph incurs an equal amount of cost/risk to the misclassification of a negative graph, i.e., all misclassifications have the same cost (In this paper, positive class and minority class are equivalent, and they both denote the class with the highest misclassification cost). The induced decision rules are commonly referred to as cost-insensitive. In real-life graph applications, the equal-cost assumption is mostly invalid (or at least too strong). Some examples are given as follows.

Biological Domains: In structure based medical diagnose [12], [13], chemical compounds active against cancer are very rare and are expected to be carefully monitored and identified. A false negative identification (i.e. predicting an active compound to be inactive) has a much more severe consequence (i.e. a higher cost) than a false positive identification (predicting an inactive compound to be active). Therefore, a false negative and a false positive are inherently different and a false negative prediction may result in the delay and wrong diagnose, leading to severe complications (or extra costs) at a later stage.

Cyber Security Domains: In intrusion detection systems, each traffic flow can be represented as a graph by presenting traffic destinations (such as IP addresses and ports) as nodes. Malicious traffics may impose threat or damage 


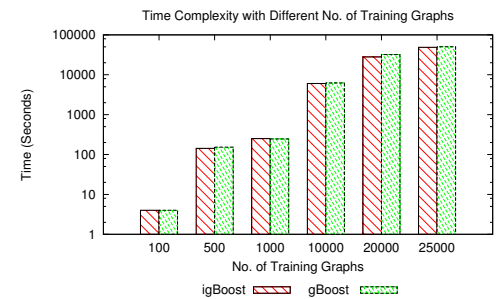

Fig. 1: Training time w.r.t. different number of graphs on NCI-1 dataset for gBoost [5] and igBoost algorithm [11]. Runtime of existing graph classification algorithms exponentially grows w.r.t. the increase of the training set size.

to computer servers, leading to severe security issues in our social life, such as private information leak or internet breakdown. Therefore, misclassification of a malicious traffic (graph) would have a much higher economic and social costs in terms of its potential impacts.

Motivated by its significance in practice, cost-sensitive learning has established itself as an active topic in data mining and machine learning areas [14], [15], [16], [17], [18], [19], [20], [21], [22], [23], [24] in the last decade. Common solutions to cost-sensitive problem includes sampling [19], decision tree modelling [25], [17], boosting [20], [21], and SVM adaptations [15], [22], [26]. However, all these methods are only dedicated to generic datasets with featurevector representation, whereas graphs do not have features immediately available and only contain nodes and their dependency structure information. Simply enumerating subgraph structures as features is clearly a suboptimal solution for cost-sensitive learning, mainly because substructure space is potentially infinite and we need a good strategy to find high quality features to help avoid misclassifications on positive classes.

Recently, an igBoost [11] algorithm has been proposed to handle imbalanced graph datasets. The igBoost approach, extended from a standard cost-insensitive graph classification algorithm gBoost [5], assigns proper weight values to different classes by taking data imbalance into consideration, so the algorithm is potentially useful to tackle the cost-sensitive learning problem for graphs. However, the loss function defined in igBoost is not cost-sensitive but only aims to minimize the misclassification errors. As a result, if the training data are separable [22], the algorithm will have limited power to enforce the cost-sensitivity learning because it only tries to separate training samples without using costs associated to different classes to tune the decisions for minimum costs. From a statistical point of view, the minimum risk could be achieved by following Bayes decision rules to predict graph samples. The objective functions in [11] is non-optimal because it simply employs some heuristic schemes, rather than implements the Bayes decision rules to minimize the conditional risk for cost-sensitive setting. In other words, the current boosting style algorithms are not targeting cost-sensitive learning problems for graph data.

\subsection{Fast Training for Large Scale Graphs}

Another challenge of existing graph classification algorithms is that they are only designed for small size graph datasets and are very inefficient to scale up to large size graph datasets. Taking existing boosting-based graph classification algorithms [11], [5] as examples, a boosting algorithm iteratively selects the most discriminative subgraphs from the graph dataset and then solves a linear programming problem for graph classification. In practice, although one may use an appropriate support value in the first step to find subgraphs, by using subgraph mining based algorithm such as gSpan [27], the linear programming solving in each iteration in the second step is a very timeconsuming process, which prevents the algorithms from scaling up to large scale graph datasets.

In Fig. 1, we report the runtime of boosting-based graph classification algorithms with respect to different numbers of training graphs. For a small number of graphs, e.g. 100 to 1000 , both gBoost [5] and igBoost [11] are relatively efficient (requiring 5 to 300 seconds for training). However, when the number of training graphs is considerably large (25,000 graphs or more), the training time for both gBoost and igBoost increase dramatically (about 50,000 seconds), and requires over 13 hours to complete the training task. As big data applications [28] are becoming increasingly popular for different domains and result in graph datasets with large volumes, finding effective boosting algorithms for large scale graph datasets is highly desired.

If we consider both cost-sensitive learning and fast graph classification as a whole, the following issues should be taken into consideration to ensure the efficiency and the effectiveness of the algorithm:

1) Cost-Sensitive Subgraph Selection: In a cost-sensitive setting, we are given a cost matrix representing misclassification costs. To ensure minimum costs for graph classification, we should take cost of individual samples into consideration to cost-sensitively select discriminative subgraphs. A cost-sensitive subgraph exploration process is, therefore, essential, but has not been addressed by existing research.

2) Model Learning for Cost-Sensitivity: For existing boosting-based graph classification algorithms, they all have non-optimal loss function, and therefore have limited capability to handle cost-sensitive problems. Alternatively, we should employ a proper loss function, which not only implements the cost-sensitive Bayes decision rule, but also approximates the Bayes risk. By doing this, the induced model will have maximum power for cost-sensitive graph classification.

3) Fast Training on Large Graph Datasets: All boosting algorithms are difficult to scale to large graphs because the optimization procedures involved in each iteration needs to resolve a large scale linear programming problem, which is typically time-consuming. We need new optimization techniques to enable fast training on large scale graph datasets.

Motivated by the above observations, we report in this 
paper, CogBoost, a fast cost-sensitive learning algorithm for graph classification. Instead of simply assigning weights to different classes, we derive a loss function which implements the Bayes decision rule and guarantees minimum risk for prediction. To identify discriminative subgraphs for cost-sensitive graph classification, we consider individual cost of each graph sample, which is completely model driven, i.e., we progressively select the most informative subgraphs based on current learned model, and the newly selected subgraph is added to current feature set to refine the classifier model. These two steps are mutually beneficial to each other. To enable fast training on large graph datasets, an advanced optimization technique, cutting plane algorithm, is derived to solving linear program efficiently. Experiments on real-word large graph datasets demonstrate CogBoost's perofrmance.

The remainder of the paper is structured as follow. Section 2 reviews related work. The problem definition and overall framework are discussed in Section 3. Section 4 reports our CogBoost algorithm for cost-sensitive graph classification. The cutting plane algorithm for fast training is reported in Section 5, followed by the time complexity analysis in Section 6. The experiments are reported in Section 7, and we conclude the paper in Section 8.

\section{Related work}

Our work is closely related to graph classification and costsensitive learning.

Graph Classification Learning and classifying graph data have drawn much attention in recent years. Because graphs involve node-edge structures whereas most existing classification methods use instance-feature representation model, the major challenge of graph classification is to transfer graphs into proper format for learning methods to train classifiers. Existing methods in the area mainly fall into two categories: (1) global similarity-based approaches [1], [4]; and (2) local subgraph based approaches [5], [7], [8]. For global similarity-based methods, graph kernels and graph embedding are used to calculate the distance between a pair of graphs, and the distance matrix can be fed into a learning algorithm, such as $k$-NN and SVM, for graph classification.

For subgraph feature based methods, the major goal is to identify significant subgraphs which can be used as signature for different classes [7], [29], [5], [9], [30]. By using subgraph features selected from the graph set, one can easily transfer graphs into a vector space so existing machine learning methods can be applied for classification [7], [29]. In [31], the authors propose a structure feature selection method to consider subgraph structural information for classification. Jin [32] proposes to extract subgraph patterns and use their co-occurrence to build classifier for graph classification. Other method [33] regards subgraph selection as combinatorial optimization problem and uses heuristic rules, in combination with frequent subgraph mining algorithm such as gSpan [27], to find subgraph features.

After obtaining subgraph features, one can also employ Boosting algorithms for graph classification [34], [5], [9],
[35]. In [9], the authors proposed to boost the subgraph decision stumps from frequent subgraphs, which means they first need to provide a minimum support to mine a set of frequent subgraphs and then utilize the function space knowledge for boosting. In contrast, gBoost [34] and its variants [5], [11] do not require a minimum support, the pruning search space relies on the effective rules derived by the authors. gBoost adopts an Adaboost procedure in [34], and it is formalized as a mathematical margin maximization problem in its latter variant [5]. The mathematical LP-boost style of algorithm in [5] demonstrated that the method is effective and converges very fast.

The above algorithms, regardless of whether they transfer graphs into a feature space or boosting directly from the weak subgraph decision stumps, are designed for costinsensitivity scenarios, i.e., they assume that all misclassifications are subject to the same cost/risk. Recently, an igBoost [11] algorithm has been proposed to deal with imbalanced graph datasets (we extend igBoost to handle dynamic data streams in [36]). igBoost assigns different weight values to different classes in order to combat class imbalance issue, which can deal with cost-sensitive problem to some extend. However, the loss function of igBoost is rather heuristic and cannot guarantee the minimization of the misclassification costs. In another words, igBoost is a sub-optimal solution to deal with cost-sensitive graph classification.

Cost-sensitive Learning Cost-sensitive learning has been extensively studied in the last decade. Approaches for costsensitive learning can be mainly distinguished into the following four categories: (1) Sampling methods [19]; (2) Decision tree approaches [25], [17]; (3) Boosting algorithms [37], [20], [21]; and (4) SVM adaptation [15], [22].

Sampling approaches [19] aim to re-weight the training samples proportional to their cost values, which can be done by over-sampling, or cost-proportionate rejection sampling. The main goal is to change the sample distributions so that any classifier can be directly used to handle cost-sensitive problems. Decision tree modelling approaches [25], [17] incorporate the costs during the tree construction, so that misclassification cost at the leaves is minimized. Boosting algorithms [37], [20], [21], such as AdaCost [37], use the misclassification costs to update the training distributions on successive boosting rounds, which has been proved to be effective to reduce the upper bound of cumulative misclassification cost of the training set. SVM adaptation [15], [22] represents a set of approaches based on SVM adaptation for cost-sensitive learning. They either shift the decision boundaries by simply adjusting the threshold of standard SVMs [26] or introduces different penalty factors $C_{1}$ and $C_{-1}$ for the positive and negative SVM slack variables during training [15]. Recently a CS-SVM algorithm [22] is proposed which utilizes an optimal hinge loss function. It is shown in [22] that CS-SVM outperforms previous approaches [15], [26].

In summary, the scope of all existing cost-sensitive methods is limited to data in vector format. In this paper, we consider the unique challenges of graph data, and propose 
a novel algorithm for cost-sensitive graph classification.

\section{Problem Definition and Overall FRAMEWORK}

Definition 1: Connected Graph: A graph is denoted as $G=(\mathcal{V}, E, \mathcal{L})$, where $V=\left\{v_{1}, \cdots, v_{n_{v}}\right\}$ is a set of vertices, $E \subseteq \mathcal{V} \times \mathcal{V}$ is a set of edges, and $\mathcal{L}$ is a labeling function assigning labels to a node or an edge. A connected graph is a graph such that there is a path between any pair of vertices.

In this paper, each labeled graph $G_{i}$ is a connected graph with a class label $y_{i} \in \mathcal{Y}=\{-1,+1\}$, i.e., we consider binary classification. If $y_{i}=+1, G_{i}$ is a positive graph, or negative otherwise.

Definition 2: Subgraph: Given two connected graphs $G=(\mathcal{V}, E, \mathcal{L})$ and $g_{i}=\left(\mathcal{V}^{\prime}, E^{\prime}, \mathcal{L}^{\prime}\right), g_{i}$ is a subgraph of $G$ (i.e $g_{i} \subseteq G$ ) if there is an injective function $\widehat{f}: \mathcal{V}^{\prime} \rightarrow \mathcal{V}$, such that $\forall(a, b) \in E^{\prime}$, we have $(\widehat{f}(a), \widehat{f}(b)) \in E, \mathcal{L}^{\prime}(a)=$ $\mathcal{L}(\widehat{f}(a)), \mathcal{L}^{\prime}(b)=\mathcal{L}(\widehat{f}(b)), \mathcal{L}^{\prime}(a, b)=\mathcal{L}(\widehat{f}(a), \widehat{f}(b))$. If $g_{i}$ is a subgraph of $G\left(g_{i} \subseteq G\right), G$ is a supergraph of $g_{i}$ $\left(G \supseteq g_{i}\right)$.

Classifier Model for Graphs: A classifier model $f(\cdot)$, which is learned from a set of training graphs $T=$ $\left\{\left(G_{1}, y_{1}\right), \cdots,\left(G_{l}, y_{l}\right)\right\}$, is a function to map a connected graph $G_{i}$ from graph space $\mathcal{G}\left(G_{i} \in \mathcal{G}\right)$ to the label space $\mathcal{Y}=\{+1,-1\}$. For cost-sensitive learning, the classifier $f(\cdot)$ is required to minimize the expected misclassification cost/risk $R=E_{G_{i}, y_{i}}\left[L\left(f\left(G_{i}\right), y_{i}\right)\right]$, where $L\left(f\left(G_{i}\right), y_{i}\right)$ is a non-negative loss function with respect to the misclassification cost. A typical loss function is as follows:

$$
L\left(f\left(G_{i}\right), y_{i}\right)=\left\{\begin{array}{rll}
0 & : & f\left(G_{i}\right)=y_{i} \\
C_{1} & : & f\left(G_{i}\right)=-1, y_{i}=1 \\
C_{-1} & : & f\left(G_{i}\right)=1, y_{i}=-1
\end{array}\right.
$$

The loss function in Eq. (1) is extended from the standard $0-1$ loss function, i.e., $L(f(\cdot), y)=I(f(\cdot) \neq y)$, where $I(\cdot)$ is an indicator function, $I(a)=1$ if $a$ holds, or $I(a)=0$ otherwise. In Eq. (1), when $C_{1}=C_{-1}=1$, the function $\left.L\left(f\left(G_{i}\right), y_{i}\right)\right)$ is cost-insensitive and degenerates to the standard 0-1 loss. For cost-sensitive learning, a false negative $\left(f\left(G_{i}\right)=-1, y_{i}=1\right)$ prediction usually incurs a larger cost than a false positive $\left(f\left(G_{i}\right)=1, y_{i}=-1\right)$ prediction, i.e., $C_{1}>C_{-1}$.

Given a set of training graphs $T=$ $\left\{\left(G_{1}, y_{1}\right), \cdots,\left(G_{l}, y_{l}\right)\right\}$, and $C_{1}$ and $C_{-1}$ for the cost of misclassification, cost-sensitive graph classification aims to build an optimal classification model $f(\cdot)$ from $T$ to minimize the expected misclassification loss (also known as risk) $R=E_{G_{i}, y_{i}}\left[L\left(f\left(G_{i}\right), y_{i}\right)\right]$. Some important notations used in the paper are listed in Table 1.

\subsection{Overall Framework}

In this paper, we propose a boosting framework for costsensitive graph classification. Our framework (Fig. 2) mainly consists of three steps.

1) Optimal Subgraph Exploration: One optimal subgraph is selected each step, and the cost-sensitive
TABLE 1: Important notations used in the paper

\begin{tabular}{ll}
\hline Symbols & Definition \\
\hline$T=\left\{G_{i}, y_{i}\right\}_{i=1, \cdots, l}$ & Training graphs with size $l$ \\
$l_{+}, l_{-}$ & Number of positive and negative graphs \\
$\boldsymbol{x}_{i}$ & Vector representation of graph $G_{i}$ \\
$\mathcal{F}=\left\{g_{1}, \cdots, g_{m}\right\}$ & The full set of subgraphs \\
$\mathcal{S}$ & Selected discriminative subgraphs \\
$\boldsymbol{w}=\left\{w_{k}\right\}_{k=1, \cdots, m}$ & Weight vectors for all subgraphs \\
$f\left(G_{i}\right), f\left(\boldsymbol{x}_{i}\right)$ & Classifier prediction on graph $G_{i}$ \\
$L\left(f\left(G_{i}\right), y_{i}\right)$ & A loss function \\
$C_{1}, C_{-}$ & Cost of positive and negative graphs, respectively \\
$\boldsymbol{\xi}=\left\{\xi_{i}\right\}_{i=1, \cdots, l}$ & Vector, slack variables for cost-sensitive learning \\
$\xi$ & Slack variable (a scalar) for cutting plane algorithm \\
$\boldsymbol{\mu}=\left\{\mu_{i}\right\}_{i=1, \cdots, l}$ & Weight vectors of training graphs \\
$C, \gamma$ & Parameters for cost-sensitive learning \\
$T_{\text {max }}$ & Maximum number of iterations \\
min_sup $_{\epsilon}$ & Mininum support for subgraph mining \\
$\epsilon$ & Cutting-plane termination threshold \\
\hline
\end{tabular}

discriminative subgraph exploration is guided by the model learnt from the previous step. The newly extracted subgraph is added to the most discriminative set $\mathcal{S}$ to enhance the learning in the next step.

2) Risk Minimization and Fast Training: A linear program is solved to achieve minimum risk based on current selected subgraphs. To enable fast training on large scale graphs, a novel cutting plane algorithm is employed.

3) Updating Graph Weights for New Iteration: After the linear program is solved, the weight values for training graphs are updated and the algorithm continues in a new iteration until the whole algorithm converges.

Next we will present our Cogboost algorithm for costsensitive graph classification and then propose a cutting plane algorithm to handle large scale graph datasets.

\section{Cost-Sensitive Learning for Graph DATA}

For graph classification, boosting [5][11] has been previously used to identify subgraphs from the training graphs as features. After that, each subgraph is regarded as a decision stump (weak classifier) to build a boosting process:

$$
\hbar_{g_{k}}\left(G_{i} ; \pi_{k}\right)=\pi_{k}\left(2 I\left(g_{k} \subseteq G_{i}\right)-1\right) ;
$$

where $\pi_{k} \in \mathcal{Y}=\{-1,+1\}$ is a parameter controlling the label of the classifier. In this paper, the weak classifier is written as $\hbar_{g_{k}}\left(G_{i}\right)$ for short.

Let $\mathcal{F}=\left\{g_{1}, \cdots, g_{m}\right\}$ be the full set of subgraphs in $T$. We can use $\mathcal{F}$ as features to represent each graph $G_{i}$ into a vector space as $\boldsymbol{x}_{i}=\left\{\hbar_{g_{1}}\left(G_{i}\right), \cdots, \hbar_{g_{m}}\left(G_{i}\right)\right\}$, with $\boldsymbol{x}_{i}^{k}=\hbar_{g_{k}}\left(G_{i}\right)$. In the following subsection, we use $G_{i}$ and $\boldsymbol{x}_{i}$ interchangeably as they are both refereed to the same graph.

The prediction rule for a graph $G_{i}$ is a linear combination of the weak classifiers:

$$
f\left(\boldsymbol{x}_{i}\right)=\boldsymbol{w}^{\boldsymbol{T}} \boldsymbol{x}_{\boldsymbol{i}}=\sum_{\left(g_{k}, \pi_{k}\right) \in \mathcal{F} \times \mathcal{Y}} w_{k} \hbar_{g_{k}}\left(G_{i}\right)
$$

where $\boldsymbol{w}=\left\{w_{k}\right\}_{k=1, \cdots, m}$ is the weight vector for all weak classifiers. The predicted class label of $\boldsymbol{x}_{i}$ is +1 (positive) if $f\left(\boldsymbol{x}_{i}\right)>0$ or -1 otherwise. 


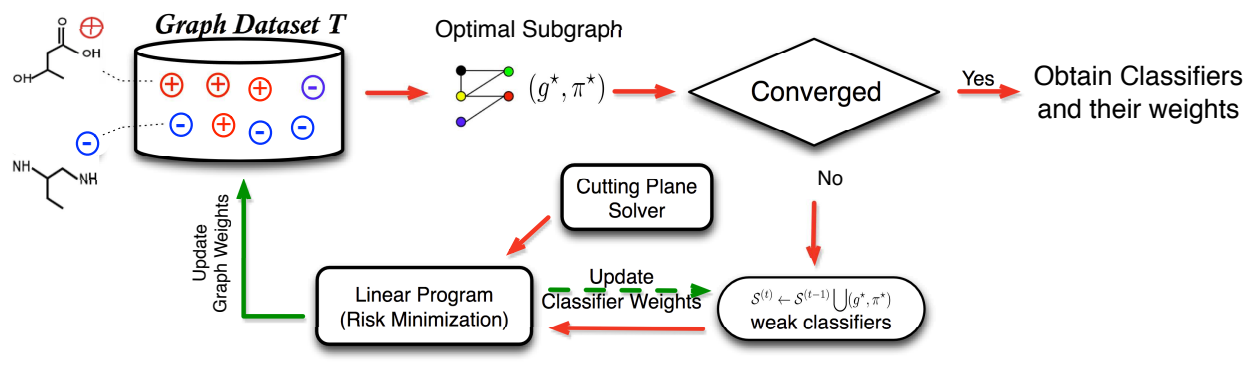

Fig. 2: The proposed fast cost-sensitive boosting for graph classification framework. In each iteration, CogBoost selects an optimal subgraph feature $\left(g^{\star}, \pi^{\star}\right)$ based on current learned model and weights of training graphs. Then $\left(g^{\star}, \pi^{\star}\right)$ is added to the current selected set $S$. Afterwards, CogBoost solves a linear programming problem to achieve cost/risk minimization. To enable fast training on large scale graph datasets, a cutting plane solver is used in this step to improve the algorithm efficiency. After solving the linear program, two sets of weights (green arrows) are updated: (1) weights for training graphs, and (2) weights for weak learners (subgraphs). The feature selection and risk minimization procedures continue until CogBoost converges or reaches the predefined number of iterations.

Similar to SVM, gBoost [5] aims to achieve minimum loss w.r.t. a standard hinge loss function $L(f, y)=\lfloor 1-$ $y f\rfloor_{+}$, where $\lfloor x\rfloor_{+}=\max (x, 0)$. igBoost [11] extends gBoost by assigning larger weight values to graphs in different classes. Both gBoost and igBoost are not optimal when dealing with cost-sensitive cases, because their loss functions do not follow the Bayes decision rules to minimize the expected risk/loss. In this section, we will first present an optimal hinge loss function, and then formalize our algorithm into a boosting paradigm.

\subsection{Optimal Cost-sensitive Loss Function}

A graph classifier $f(\cdot)$ maps a graph $G_{i}$ to a class label $y_{i} \in\{-1,1\}$. Assume graphs and class labels are drawn from probability distribution $P_{\mathcal{G}}\left(G_{i}\right)$ and $P_{\mathcal{Y}}\left(y_{i}\right)$, respectively. Given a non-negative loss function $L\left(f\left(G_{i}\right), y_{i}\right)$, the classifier $f\left(G_{i}\right)$ is optimal if it minimizes the loss/risk $R=E_{G_{i}, y_{i}}\left[L\left(f\left(G_{i}\right), y_{i}\right)\right]$. Let $\eta=P_{\mathcal{Y} \mid \mathcal{G}}\left(1 \mid G_{i}\right)$ be the probability for $G_{i}$ being 1 , from a Bayes decision rule point of view, this is equivalent to minimize the conditional risk.

$$
\begin{aligned}
E_{\mathcal{Y} \mid \mathcal{G}}\left(L\left(f\left(G_{i}\right), y_{i}\right) \mid G=G_{i}\right) & =\eta L\left(f\left(G_{i}\right), 1\right) \\
& +(1-\eta) L\left(f\left(G_{i}\right),-1\right)
\end{aligned}
$$

The loss function in Eq. (1) is a Bayes consistent loss function [38], i.e., it implements the Bayes decision rule to achieve minimum conditional risk (Eq. (4)). This suggests that ideally Eq. (1) can be used to design some costsensitive algorithms for minimizing conditional risk. However, Eq. (1) is extended by a 0-1 loss function. Minimizing the $0-1$ loss is computationally expensive because it is not convex. State of the art algorithms usually use surrogate loss functions to approximate the 0-1 loss (e.g., SVM and gBoost [5] employ hinge loss). The hinge loss induced SVM algorithms enforces maximum margins between the support vectors and the hyper-planes, which can achieve good classification performance.

A recent work on SVM [22] theoretically suggests that the standard hinge loss can be extended to be cost-sensitive, by setting the loss function $L\left(f\left(G_{i}\right), y_{i}\right)$ as follows:

$L\left(f\left(G_{i}\right), y_{i}\right)=\left\{\begin{aligned}\left\lfloor C_{1}-C_{1} \cdot f\left(G_{i}\right)\right\rfloor_{+} & : \quad y_{i}=1 \\ \left\lfloor 1+\left(2 C_{-1}-1\right) \cdot f\left(G_{i}\right)\right\rfloor_{+} & : \quad y_{i}=-1\end{aligned}\right.$
It is proved in [22] that the new hinge loss function Eq. (5) also implements the Bayes decision rule. Additionally, employing Eq. (5) also enjoys the merit of maximum margin principal for classification. The standard hinge loss and its cost-sensitive hinge loss is illustrated in Fig. 3. They have different explanations with respect to the loss and the margins (distance to the hyperplane from support vectors). Specifically, for standard hinge loss (Fig. 3. (A)), the positive and negative class both have equal margins (unit margins); and for cost-sensitive hinge loss (Fig. 3. (B) ), the negative class has a much smaller margin when the positive class still have a unit margin. As shown in Fig. 3. (C), the margins for positive and negative classes are uneven when cost-sensitive hinge loss function Eq. (5) is utilized in a SVM formulation.

Note that the loss function employed in igBoost [11] is heuristically adapted from standard hinge loss, which does not necessarily follow the Bayes decision rule. In other words, it is a sub-optimal loss function for cost-sensitive learning. In the following subsection, we will use the costsensitive hinge loss function in Eq. (5), and re-formulate it into a linear program boosting framework.

\subsection{Cost-Sensitive Formulation for Graphs}

Motivated by the optimal loss function in Eq. (5), we formalize our learning task as the following regularized risk minimization problem:

$$
\begin{array}{ll}
\min _{\boldsymbol{w}} & \|\boldsymbol{w}\|+\frac{C}{l}\left\{\sum_{\left\{i \mid y_{i}=1\right\}} L\left(f\left(\boldsymbol{x}_{i}\right), 1\right)+\sum_{\left\{j \mid y_{j}=-1\right\}} L\left(f\left(\boldsymbol{x}_{j}\right),-1\right)\right\} \\
& \text { s.t. } \quad \boldsymbol{w} \succeq 0
\end{array}
$$

In Eq.(6), we enforce the weight for each subgraph to be positive, i.e., $\boldsymbol{w} \succeq 0$. We also impose 1-norm regularization on $\boldsymbol{w}$ (i.e., $\|\boldsymbol{w}\|$ ), which will favor sparse solutions with many variables being exactly 0 . This strategy is similar to the problem of LASSO for variable shrinking [39]. And we use $i$ and $j$ to index the positive and negative training graphs, respectively. $C$ is a parameter to tradeoff the regularization term and loss term. The objective 

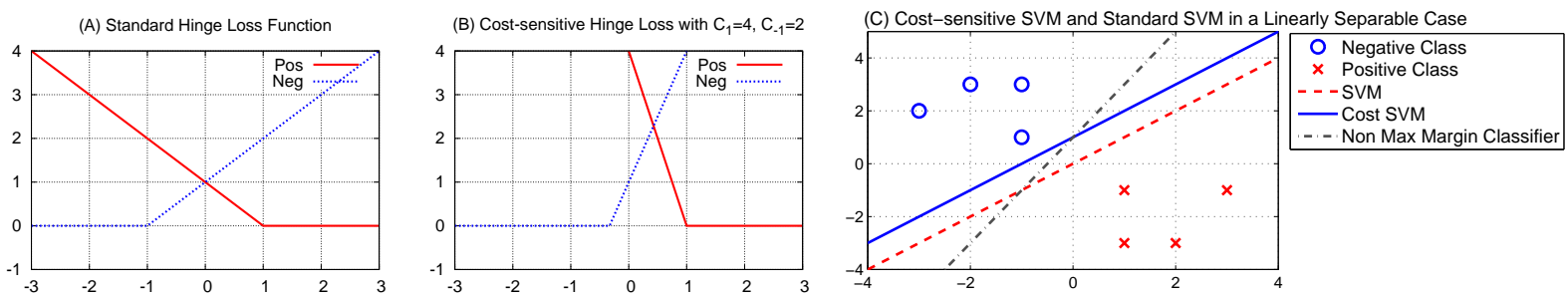

Fig. 3: Different loss functions and formulations with respect to support vector machines (SVMs): (A) Standard Hinge Loss, (B) Cost-sensitive Hinge Loss with $C_{1}=4$ and $C_{-1}=2$, and (C) Different SVM formulations with Standard Hinge Loss and Cost-sensitive Hinge Loss (cf.[22]).

function in Eq.(6) can be reformulated as follows:

$$
\begin{array}{ll}
\min _{\boldsymbol{w}, \boldsymbol{\xi}} & \|\boldsymbol{w}\|+\frac{C}{l}\left\{C_{1} \sum_{\left\{i \mid y_{i}=1\right\}} \xi_{i}+\gamma \sum_{\left\{j \mid y_{j}=-1\right\}} \xi_{j}\right\} \\
\text { s.t. } & f\left(\boldsymbol{x}_{i}\right) \geq 1-\xi_{i}, y_{i}=1 \\
& f\left(\boldsymbol{x}_{j}\right) \leq-\frac{1}{\gamma}+\xi_{j}, y_{j}=-1 \\
& f\left(\boldsymbol{x}_{i}\right)=\sum_{k=1}^{m} w_{k} \cdot \hbar_{g_{k}}\left(G_{i}\right) \\
& \boldsymbol{w} \succeq 0, \boldsymbol{\xi} \succeq 0, \gamma=2 C_{-1}-1,
\end{array}
$$

In Eq.(7), $\xi_{i}$ and $\xi_{j}$ are slack variables concerning the loss of misclassifying a positive and a negative graph, respectively. In this case, cost-sensitivity is controlled by $C_{1}$ and $\gamma$, which impose a smaller margin on negative examples than positive examples (In Fig. 3. (B) and (C), for an example with $C_{1}=4$, and $\gamma=2 C_{-1}-1=3$, the margin for negative example is $\frac{1}{\gamma}=\frac{1}{3}$ ). As suggested in [38], we can set $\gamma$ as a parameter subject to $1 \leq \gamma \leq C_{1}$ instead of a fixed value $\left(2 C_{-1}-1\right)$ to achieve better classification.

Solving objective function in Eq. (7) requires a complete set of subgraph features (i.e., represent $G_{i}$ as $\boldsymbol{x}_{i}=$ $\left.\left\{\hbar_{g_{1}}\left(G_{i}\right), \cdots, \hbar_{g_{m}}\left(G_{i}\right)\right\}\right)$, which are unavailable unless we enumerate the whole subgraph space in advance. In practice, this is likely impossible because the whole subgraph set is very large and possibly infinite. In the following subsection, we will transfer this formulation to its Lagrange dual problem and use a boosting algorithm to solve it in an iterative way.

\subsection{Boosting for Cost-sensitive Learning on Graphs}

The Lagrange dual of a problem usually provides additional insights to the original (primal) problem. The dual problem of Eq.(7) is ${ }^{1}$

$$
\begin{array}{ll}
\max _{\boldsymbol{\mu}} & \sum_{i=1}^{l_{+}} \mu_{i}+\frac{1}{\gamma} \sum_{j=1}^{l_{-}} \mu_{j} \\
\text { s.t. } & \sum_{i=1}^{l_{+}} \mu_{i} \hbar_{g_{k}}\left(G_{i}\right)-\sum_{j=1}^{l_{-}} \mu_{j} \hbar_{g_{k}}\left(G_{j}\right) \leq 1, \forall g_{k} \in \mathcal{F} \\
& 0 \leq \mu_{i} \leq \frac{C C_{-1}}{l}, i=1, \cdots, l^{+} \\
& 0 \leq \mu_{j} \leq \frac{\gamma C}{l}, \quad j=1, \cdots, l_{-}
\end{array}
$$

where $l_{+}$and $l_{-}$indicate the number of graphs in positive and negative sets $\left(l=l_{+}+l_{-}\right)$, respectively. While solving the primal problem in Eq.(7) returns a vector $\boldsymbol{w}$ indicating

1. The derivation from the primal problem Eq.(7) to dual problem Eq.(8) is shown in Appendix A.1. the weights of each subgraphs, the dual problem in Eq. (8) will produce a vector $\boldsymbol{\mu}=\left\{\mu_{i}\right\}_{i=1, \cdots, l}$. Nevertheless, Eq.(7) and Eq.(8) will generate the same objective values. Insights of Dual Problem (1) The solution $\left\{\mu_{i}\right\}_{i=1, \cdots, l}$ can be interpreted as the weight values of graphs in order to achieve minimum loss. (2) Each constraint $\sum_{i=1}^{l_{+}} \mu_{i} h_{g_{k}}\left(G_{i}\right)-\sum_{j=1}^{l_{-}} \mu_{j} h_{g_{k}}\left(G_{j}\right) \leq 1$ in Eq. (8) indicates a subgraph pattern $g_{k}$ over the whole training graphs. It provides a natural metric to assess the cost-sensitive discriminative power of a subgraph.

Definition 3: Cost-sensitive Discriminative Score: For a subgraph decision stump $\hbar_{g_{k}}\left(G_{i}\right)$, its cost-sensitive discriminative score over the whole training graphs is:

$$
\Theta\left(g_{k}, \pi_{k}\right)=\sum_{i=1}^{l_{+}} \mu_{i} \hbar_{g_{k}}\left(G_{i}\right)-\sum_{j=1}^{l_{-}} \mu_{j} \hbar_{g_{k}}\left(G_{j}\right)
$$

Eq.(8) requires discriminative scores for all subgraphs $\leq 1$, which latter will serve as an termination condition of our iterative algorithm.

Linear Program Boosting Framework Because we do not have a predefined feature set $\mathcal{F}$ in advance, we cannot solve Eq.(7) or Eq.(8). Therefore, we propose to use column generation $(C G)$ techniques [40] to solve the objective function (Eq. (7)). The idea of CG is to begin with an empty feature set $\mathcal{S}$, and iteratively select and add one feature/column to $\mathcal{S}$ which violates the constraint in the dual problem (Eq. (8)) mostly. After $\mathcal{S}$ is updated, CG resolve the primal problem Eq.(7). This procedure continues until no more subgraph violating the constraint in (Eq.(8)).

Our cost-sensitive graph boosting framework is illustrated in Algorithm 1. CogBoost iteratively selects the most discriminative subgraph $\left(g^{\star}, \pi^{\star}\right)$ at each round (step 4). If the current optimal pattern no longer violates the constraint or it has reached the maximum number of iterations $T_{\max }$, the iteration process stops (steps 5-6). Because in the last few iterations, the optimal value only changes subtlety, we add a small value $\Delta$ to relax the stopping condition (typically, we use $\Delta=0.01$ in our experiments). In step 8 , we solve the linear programming problem based on the selected subgraphs to recalculate two set of weights: (1) $w^{(t)}$, the weights for subgraph decision stumps in $\mathcal{S}$; and (2) $\boldsymbol{\mu}^{(t)}$, the weights of training graph for optimal subgraph mining in the next round, which can be obtained from the Lagrange multipliers of the primal problem. Once the algorithm converges or the number of maximum iteration is 

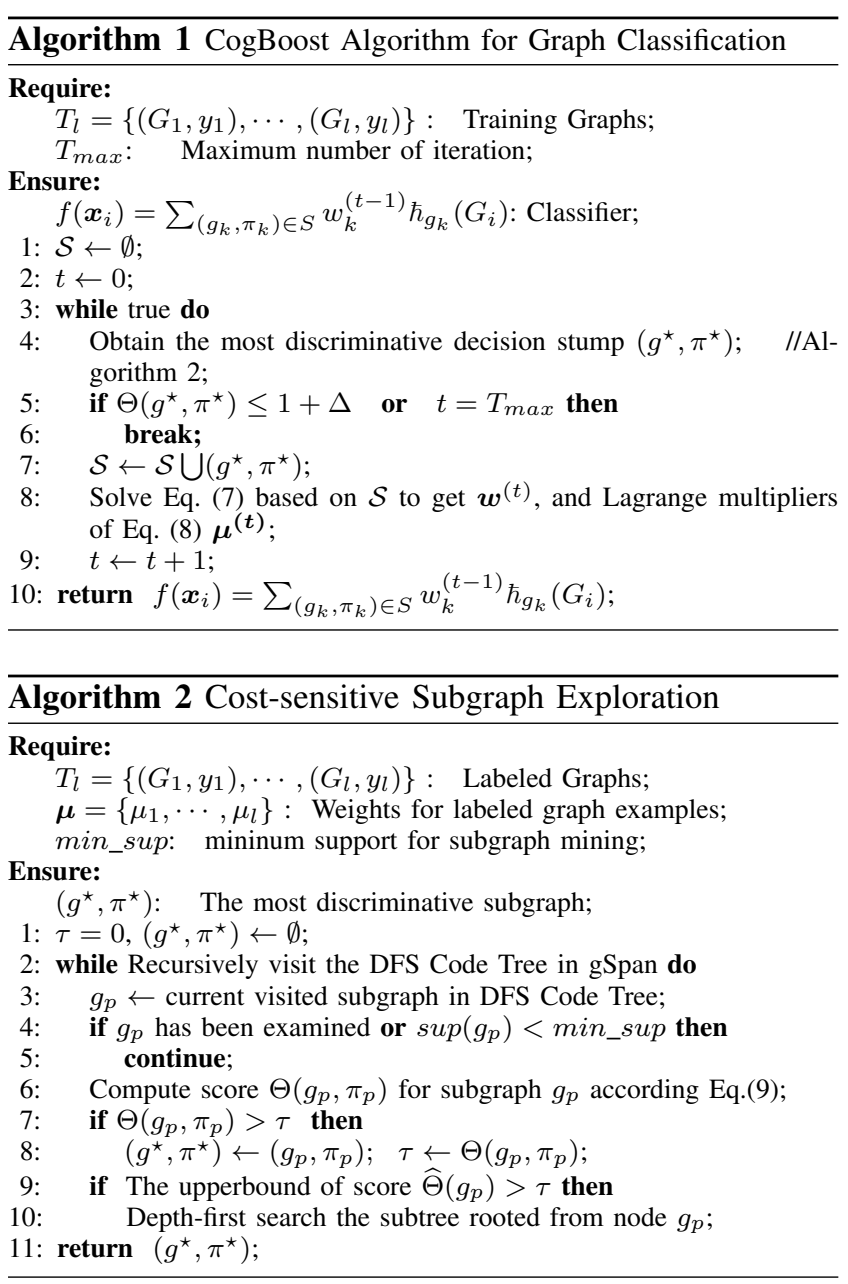

reached, CogBoost returns the final classifier model $f\left(\boldsymbol{x}_{i}\right)$ in step 10 .

\subsection{Cost-sensitive Subgraph Exploration}

To learn the classification model, we need to find the most discriminative subgraph which considers each training graph's weight in each step (step 4 in Algorithm 1). The subgraph exploration is completely model driven, i.e., we select a subgraph which violates the constraint in Eq.(8) mostly. Based on the definition of discriminative score in Eq.(9), we need to perform a weighted subgraph mining over training graphs.

In CogBoost, we employ a Depth-First-Search (DFS) based algorithm gSpan [27] to enumerate subgraphs. The key idea of gSpan is that each subgraph has a unique DFS Code, which is defined by its lexicographic order of the discovery time during the search process. Two subgraphs are isomorphism iff they have the same minimum DFS Code. By employing a depth first search strategy on the DFS Code tree (where each node is a subgraph), gSpan can enumerate all frequent subgraphs efficiently. To speed up the enumeration, we further employ a branch-and-bound scheme to prune the search space of DFS Code tree by utilizing an upper bound of discriminative score [5] for each subgraph pattern.
Our subgraph mining algorithm is listed in Algorithm 2. The minimum value $\tau$ and optimal subgraph $\left(g^{\star}, \pi^{\star}\right)$ are initialized in step 1 . We prune out duplicated subgraph features or subgraph with low support $(\sup (\cdot)$ returns the support of a subgraph) in step 4-5, and compute the discriminative score $\Theta\left(g_{p}, \pi_{p}\right)$ for $g_{p}$ in step 6. If $\Theta\left(g_{p}, \pi_{p}\right)$ is larger than $\tau$, we update the optimal subgraph in step 8 . We use an branch-and-bound pruning rule in [5] to prune the search space in steps 9-10. Finally, the optimal subgraph pattern $\left(g^{\star}, \pi^{\star}\right)$ is returned in step 11 .

\section{Fast Training for large Scale GRAPHS}

For CogBoost algorithm, it needs to iteratively mine an optimal subgraph (step 4 of Algorithm 1) and solve a linear problem (step 8 of Algorithm 1). To enable fast training for large scale graph datasets, for step 4 we can set a proper support and use some heuristic techniques, such as reusing the search space during the enumeration of subgraphs rather than re-mining subgraph from scratch, just as [5] does. For step 8 , in this section, we derive a cutting plane algorithm to speed up the training process.

\subsection{From $l$-Slacks to 1-Slack Formulation}

Eq.(7) in step 8 of Algorithm 1 has $l=l_{+}+l_{-}$slack variables $\xi_{i}$ and $\xi_{j}$, inspired by the techniques used in the SVM formulation [41], we propose to solve it efficiently by reducing the number of slack variables as follows,

$$
\begin{aligned}
& \min _{\boldsymbol{w}, \xi} \quad\|\boldsymbol{w}\|+C \xi \\
& \text { s.t. } \quad \forall \boldsymbol{w}, \xi \in\{0,1\}^{l}, \quad \frac{1}{l} \boldsymbol{w}^{T}\left\{C_{1} \sum_{y_{i}=1} c_{i} \boldsymbol{x}_{i}-\gamma \sum_{y_{j}=-1} c_{j} \boldsymbol{x}_{j}\right\} \\
& \geq \frac{1}{l}\left\{C_{1} \sum_{y_{i}=1} c_{i}+\sum_{y_{j}=1} c_{j}\right\}-\xi, \\
& \boldsymbol{w} \succeq 0, \xi \geq 0 .
\end{aligned}
$$

The above formulation only has one slack variable $\xi$, which can be proved to be equal to Eq.(7) ${ }^{2}$, with $\xi=\left\{C_{1} \sum_{\left\{i \mid y_{i}=1\right\}} \xi_{i}+\gamma \sum_{\left\{j \mid y_{j}=-1\right\}} \xi_{j}\right\} / l$. Note that although Eq.(10) has $2^{l}$ constraints in total, such a formulation can be solved by cutting plane algorithm in a linear time by iteratively selecting a small number of most violated constraints (Cutting Planes). This leads to an efficient solution to the optimization, so our algorithm can effectively scale to large datasets.

The dual of $l$-slack formulation in Eq.(8) provides solution $\boldsymbol{\mu}$ which can interpret the graph weights for subgraph mining in the next iteration. To establish the same relationship between the new objective function Eq.(10) and the graph weights $\boldsymbol{\mu}$, we also refer to its dual problem, which is given as follows ${ }^{3}$ :

$$
\begin{array}{ll}
\max _{\lambda_{c}} & \frac{c_{1}}{l} \sum_{c} \lambda_{c} \sum_{i} c_{i}+\frac{1}{l} \sum_{c} \lambda_{c} \sum_{i} c_{j} \\
\text { s.t. } & \frac{C_{1}}{l} \sum_{c} \lambda_{c} \sum_{i} c_{i} \boldsymbol{x}_{i}^{k}-\frac{\gamma}{l} \sum_{c} \lambda_{c} \sum_{j} c_{j} \boldsymbol{x}_{j}^{k} \leq 1, \forall k \\
& 0 \leq \sum_{c} \lambda_{c} \leq C .
\end{array}
$$


Comparing the the dual problems of Eq.(8) and Eq.(11), they are identical if:

$$
\mu_{i \mid y_{i}=1}=\frac{C_{1}}{l} \sum_{c} \lambda_{c} c_{i} ; \mu_{j \mid y_{j}=-1}=\frac{\gamma}{l} \sum_{c} \lambda_{c} c_{j}
$$

\subsection{Cutting-plane Algorithm for Fast Training}

The basic idea of cutting-plane algorithm is similar to the column generation algorithm, or it can be regarded as a row generation algorithm (each constrain in Eq.(11) is a row). Instead of considering all constrains (rows) as a whole, our cutting-plane algorithm considers only the most violated constraint (row) each time. The selected violated constraints form a working set $\mathcal{W}$. And it utilizes an iterative procedure to solve the problem. By doing this, the linear program can be solve efficiently.

Our detailed cutting plane algorithm is shown in Algorithm 3. Initially, the working set $\mathcal{W}$ is an empty set in step 1 . In each iteration, we solve the optimization problem based on current working set $\mathcal{W}$ in step 3 ( $\boldsymbol{w}=0$ and $\xi=0$ for the first iteration). Steps 4-6 find the most violated constraint, which is determined by the cost-sensitive loss function in Eq.(5). Step 9 adds the current most constraint to the working set. The iteration continues until it reaches the convergence (steps 7-8). Also, we add a small constant $\epsilon$ (In our experiments, we set $\epsilon=0.01$ as default value) to enable early termination of iterations.

Our cutting plane algorithm can always return an $\epsilon$ tolerance accurate solution (approximate the solution of Eq. (7) very well). It is efficient because each time we solve a linear program in a small working set, the cutting plan algorithm is independent of the number of sample size. This essentially ensures that our solutions can scale to very large scale graph datasets.

\section{Time Complexity Analysis: Theoret- ical Aspect and Practice}

The time complexity of CogBoost includes two major components: (1) mining a cost-sensitive discriminative subgraphs $\mathcal{O}(P(l))$ (step 4 of Algorithm 1), and (2) solving a linear program problem $\mathcal{O}(Q(l)$ ) (step 8 of Algorithm 1 ), where $P$ and $Q$ are functions for mining subgraph and solving LP problem of size $l$. For subgraph mining, CogBoost employs a gSpan based algorithm (Algorithm 2) for subgraph enumeration in the first iteration $(\mathcal{O}(P(l)))$, and re-uses the search space [5] of the first iteration $(\mathcal{O}(\bar{P}(l)))$. Because re-using search space can significantly reduce the mining time, we have $\mathcal{O}(\bar{P}(l)) \ll \mathcal{O}(P(l))$. Suppose the number of iterations of CogBoost (Algorithm 1 ) is $T_{\max }$, the total time complexity of CogBoost is:

$$
\mathcal{O}=\mathcal{O}(P(l))+\left(T_{\max }-1\right) \mathcal{O}(\bar{P}(l))+T_{\max } \mathcal{O}(Q(l))
$$

\subsection{Time complexity of Subgraph Mining}

Theoretical Aspect: Intuitively, because the subgraph space is infinitely large, the time complexity for subgraph

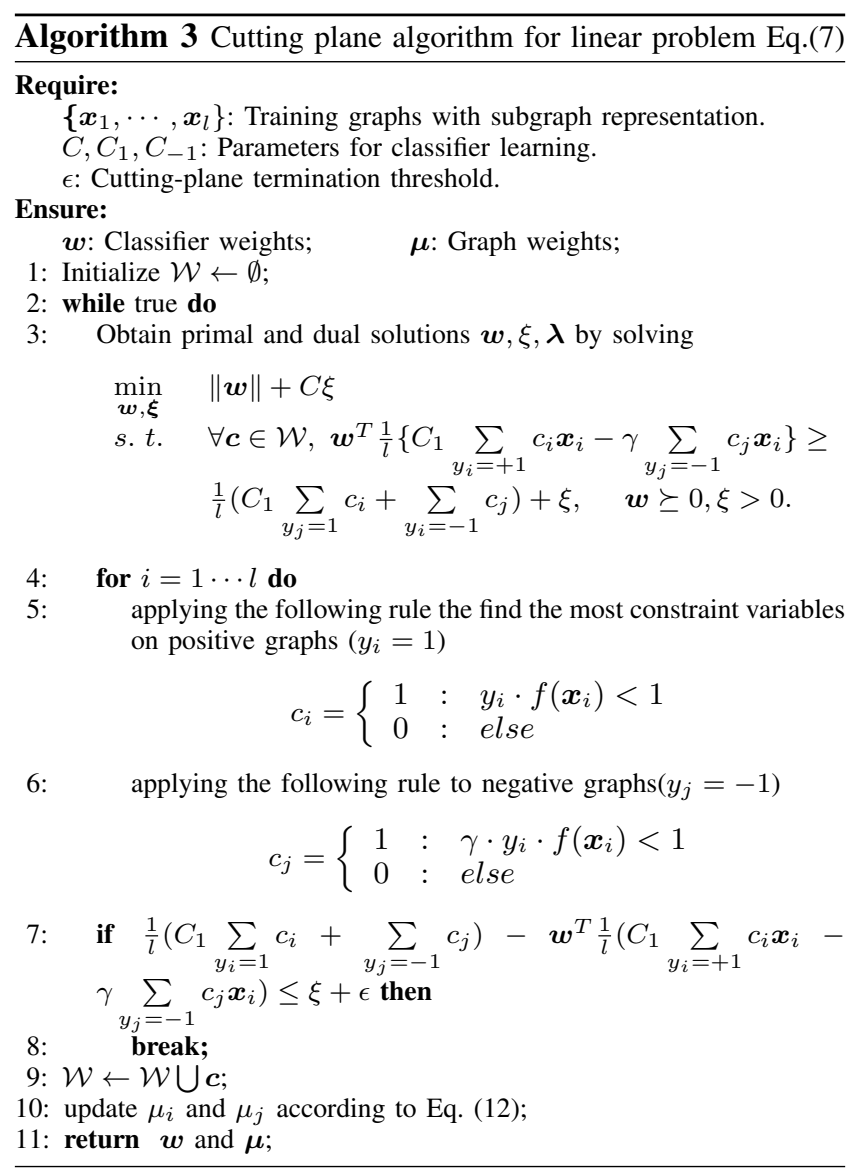

mining is NP-hard, and $O(P(l))$ for subgraph mining is inevitable for graph classification. Thus all existing subgraph feature selection algorithms for graph classification [5], [7], [33] derive some upper-bounds to prune the search space. In CogBoost, we incorporate the upper-bound in [5] and the support threshold min_sup to reduce the sugraph space. It is worth noting that $\operatorname{CogBoost}$ can still function property even though users do not specify the min_sup value for subgraph mining. If min_sup were not specified, CogBoost will only rely on the upper-bound in [5] to prune the search space.

Practice: In practice, we observe that when the data set is considerable large (e.g., 40,000 chemical compounds or more), setting a support threshold min_sup $=5 \%$ can significantly speed up the mining progress. However, setting a threshold may incur missing of discriminative subgraphs because some infrequent subgraphs are not checked. Accordingly, we suggest removing the min_sup threshold for small graph dataset while setting a proper support for large datasets. The proper support value depends on the domains of applications. For instance, when the average number of nodes and edges of the graph dataset are large, a larger support (5-10\%) is preferred. On the other hand, if the average number of nodes and edges are small, a small support (about 1\%-3\%) is a good choice. For real-world applications, it is useful to first check the statistics of the graph samples before carrying out the graph classification tasks. 
TABLE 2: Graph Datasets Used in the Experiments

\begin{tabular}{cccccc}
\hline Datasets & \#Pos & \#Total & \#Nodes & \#Edges & Descriptions \\
\hline NCI-1 & 1,793 & 37,349 & 26 & 28 & Lung Cancer \\
NCI-33 & 1,467 & 37,022 & 26 & 28 & Melanoma \\
NCI-41 & 1,350 & 25,336 & 27 & 29 & Prostate Cancer \\
NCI-47 & 1,735 & 37,298 & 26 & 28 & Central Nerve \\
NCI-81 & 2,081 & 37,549 & 26 & 28 & Colon Cancer \\
NCI-83 & 1,959 & 25,550 & 27 & 29 & Breast Cancer \\
NCI-109 & 1,773 & 37,518 & 26 & 28 & Ovarian \\
NCI-123 & 2,715 & 36,903 & 26 & 28 & Leukemia \\
NCI-145 & 1,641 & 37,041 & 26 & 28 & Renal Cancer \\
\hline Twitter & 66,458 & 140,949 & 4 & 5 & Sentiment \\
\hline
\end{tabular}

\subsection{Time complexity of LP Solving}

Theoretical Aspect: For LP problem solving, Eq. (7) is solvable in polynomial time $O(Q(l))=\mathcal{O}\left(l^{k}\right)$ with some constant $k$ [42]. In other words, gBoost [5] and igBoost [11] needs polynomial time for this step. By using cutting plane algorithm, the time complexity would be $O(Q(l))=\mathcal{O}(s l)$, where $s$ is the number of non-zeros features in the original problem (please refer to [41] for detailed analysis). Therefore, CogBoost can significantly reduce the runtime when the graph sample size $l$ is large. In Section 7, our experiments will soon demonstrate that the improvement of LP problem solving without using cutting plan algorithm is marginal.

Practice: The cutting plane algorithm uses a working set $\mathcal{W}$ (in Algorithm 3), $\mathcal{W}$ overlaps significantly during two consecutive iteration of Algorithm 1. Therefore, in our implementations, we re-use top 200 most violated constrains in $\mathcal{W}$ in the previous iteration, which can significantly improve the algorithm efficiency. In practice, the classifier weights $\boldsymbol{w}$ in two consecutive iterations may be very close to each other, one can also use the warm-start technique (using $\boldsymbol{w}$ in previous iteration as initial value for linear problem solving) to speed up the learning process.

\section{EXPERIMENTS}

In this section, we evaluate CogBoost in terms of its average misclassification cost (or average cost) and runtime performance. The average cost is calculated by using the total misclassification costs divided by the number of test instances. The lower the average costs, the better the algorithm performance is. The runtime performance is evaluated based on the actual runtime of the algorithm.

\subsection{Experimental Settings}

Two types of real-life datasets, NCI chemical compounds and Twitter graphs, are used in our experiments. Table 2 summaries the statistics of the two benchmark datasets.

NCI Graph Datasets are commonly used as the benchmark for graph classification. In our experiments, we download nine NCI datasets from PubChem ${ }^{4}$. Each NCI dataset belongs to a bioassay task for anticancer activity prediction, where each chemical compound is represented as a graph,

4. http://pubchem.ncbi.nlm.nih.gov with atoms representing nodes and bonds as edges. A chemical compound is positive if it is active against the corresponding cancer, or negative otherwise.

Table 2 summarizes the NCI graph data used in our experiments. We have removed disconnected graphs and graphs with unexpected atoms (some graphs have atoms represented as '**) in the original graphs. Columns 2-3 show the the number of positive and total number of graphs in each dataset, and Columns 4-5 indicate the average number of nodes and edges in each dataset, respectively.

Stanford Twitter Graphs are extracted from twitter sentiment classification ${ }^{5}$. Because of the inherently short and sparse nature, twitter sentiment analysis (i.e., predicting whether a tweet reflects a positive or a negative feeling) is a difficult task. To build a graph dataset, we represent each tweet as a graph by using tweet content, with nodes in each graph denoting the terms and/or smiley symbols (e.g, :-D and :-P) and edges indicating the co-occurrence relationship between two words or symbols in each tweet. To ensure the quality of the graph, we only use tweets containing 20 or more words. In our experiments, we use tweets from April 6 to June 16 to generate 140,949 graphs (in a chronological order). Note that this dataset has been used for simulated graph stream classification in our previous study [36]. In this paper, we aggregate all graphs as one dataset without considering their temporal order.

Baselines We compare our proposed CogBoost algorithm with the following baseline algorithms.

- gBoost [5] is a state-of-the-art boosting method, which has demonstrated good performance for graph classification.

- igBoost [11] extends gBoost to handle imbalanced graph datasets. The weight of a minority (positive) graph is assigned with a $\beta$ times higher weight value than a majority (negative) graph.

- Fre+CSVM first mines a set of frequent subgraphs (with minimum support 3\%) from the entire graph dataset, and selects the top- $K$ most frequent subgraphs as features. Afterwards, each graph dataset is transferred into vector format by checking the existence of selected subgraphs in the original graph datasets. Finally, the cost-sensitive support vector machine algorithm [15], [18] is applied to the transferred vectors.

- gSemi+CSVM ${ }^{6}$ employs a gSemi [7] algorithm to mine top- $K$ discriminative subgraphs from the entire graph dataset, and then transfers the original graph database into vectors. Similar to Fre+CSVM, the costsensitive SVM algorithm [18] is used to learn a model from the transferred vectors.

To validate the effectiveness of the cutting plane solver in our CogBoost algorithm for large scale graphs, we implement two variants of CogBoost,

5. http://jmgomezhidalgo.blogspot.com.au/2013/01/a-list-of-datasetsfor-opinion-mining.html

6. We encounter an out-of-memory error for gSemi+CSVM algorithm on Twitter graph dataset, because gSemi algorithm [7] needs to do matrix calculation to select subgraphs. Java fails to create such a large "double" matrix (about 100,000*100,000) 
- CogBoost-a: This variant discards the cutting plane module and solves the linear program of Eq.(7) directly. In other words, it uses all $l$ slack variables in total.

- CogBoost-1: The CogBoost-1 utilizes the cutting plane module to solve the linear program (Eq.(10)) for large scale graphs, i.e., it has only one (i.e. 1) slack variable each time.

For each graph dataset, we randomly split it into two subsets. The training set consists of $70 \%$ of the graph dataset, and the rest is used as the test set. The results reported in the paper are based on the average of a number of repetitions. Note that for gBoost [5] and igBoost [11], the previous studies only validate their performance using a rather small number of graphs (from several hundreds to several thousands graphs), whereas in our experiments, our training data is much larger.

Parameter Settings For fair comparisons, the default misclassification cost for positive graphs is set as $C_{1}=20$ for NCI graphs, which is actually the approximated imbalanced ratio $\left(\frac{|N e g|}{\mid P o s} \mid\right)$ of these graph datasets. For Twitter graphs, a large $C_{1}$ will result in that all graphs are classified in one class for all algorithm. To avoid this case, we set the default value $C_{1}=3$. For all experiments, the cost for negative graphs is always set as $C_{-1}=1$ for all datasets. As suggested in [38], we selected the best parameter $\gamma$ instead of fixing it to $2 C_{-1}-1$ for CogBoost algorithm. For igBoost, we set $\beta=C_{1}$, so positive class graphs have a weight value $\beta$ times higher than negative graphs. The regularization parameter in our algorithm is $C$, and $D=1 / v$ for gBoost and igBoost. To make them comparable, we vary $C$ from $\{0.1,1,10,100,1000,10000\}$, and $v$ from $\{0.01,0.2,0.4,0.6,0.8,1.0\}$. These candidate values are set according to the property of each algorithm. min_sup is set to 5\% for NCI graphs and $0.5 \%$ for Twitter graphs.

Because both igBoost and gBoost require over 10 hours to complete a classification task, it is impractical to select the best parameters for each algorithm on the whole training graphs on each dataset. Therefore, we select the parameters for each algorithm which achieves the minimum misclassification cost over a sample of 5000 training graphs on each dataset. Then we train the classifiers with these selected parameters on the whole training graphs. For Fre+CSVM and gSemi+CSVM, the number of most informative subgrahps $K$ is always equal to $T_{\max }$ employed into another boosting algorithm, i.e., we ensure that all algorithms use the same number of features for graph classification.

Unless specified otherwise, other parameters for our algorithm is set as follows: $T_{\max }=50$ and $\epsilon=0.01$.

All our algorithms are implemented using a Java package MoSS ${ }^{7}$ and Matlab toolbox $\mathrm{CVX}^{8}$. MoSS provides a framework for frequent subgraph mining, and CVX serves as a module for solving linear programs. JavaBuilder provided by Matlab bridges MoSS and CVX into a united

7. http://www.borgelt.net/moss.html

8. http://cvxr.com/cvx/

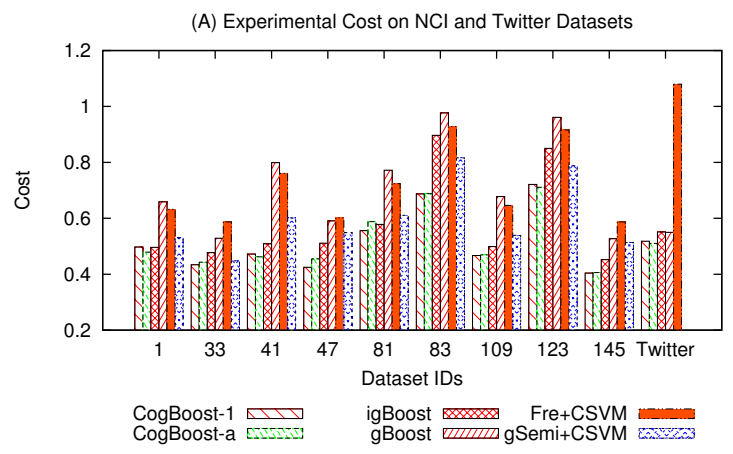

(B) Time Complexity on $\mathrm{NCl}$ and Twitter Datasets

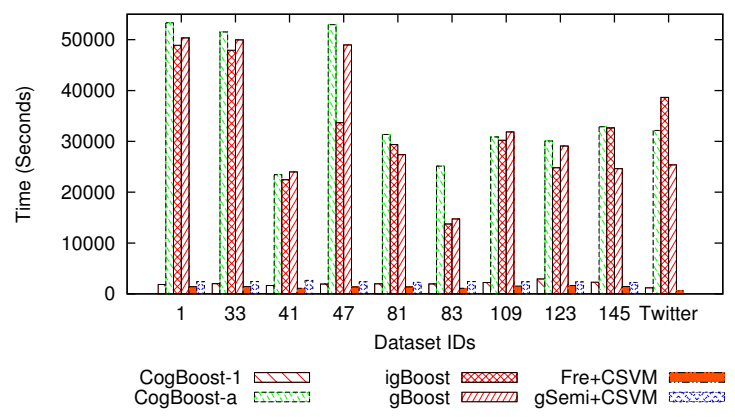

Fig. 4: Experimental Results. (A) Average cost, (B) Time Complexity.

framework. All our experiments are conducted on a cluster node of Red Hat OS with 12 processors (X5690 @ $3.47 \mathrm{GHz}$ ) and 48GB memory.

\subsection{Experimental Results}

In this subsection, we evaluate the effectiveness of CogBoost for cost-sensitive learning and fast cutting-plane training in terms of average cost and runtime performance. The experimental results for NCI and Twitter graphs under default parameter settings are illustrated in Fig. 4.

Average Cost For average cost, Fig. 4. (A) demonstrates that gBoost has the worst performance on 5 out of 10 datasets (i.e. the largest average cost). This is mainly because gBoost is a cost-insensitive algorithm, which considers that all training graphs are equally important in terms of their costs. As a result, gBoost fails to leverage the costs of graph samples to discover subgraph features mostly discriminative for differentiating graphs in the positive class, leading to deteriorated classification performance.

For igBoost, Fre+CSVM, and gSemi+CSVM, all of them have a mechanism to assign weight values to different classes. Fig. 4. (A) shows that igBoost outperforms Fre+CSVM and gSemi+CSVM, which is mainly attributed to igBoost's integration of discriminative subgraph selection and classifier learning for graph classification. For Fre+CSVM and gSemi+CSVM, they decompose subgraph selection and classifier learning into two separated steps, without integrating them to gain mutual benefits, i.e., the subgraphs selected by frequency and gSemi score [7] may not be a good feature set for SVM learning. As a result, Fre+CSVM and gSemi+CSVM are inferior to igBoost. 
This is, in fact, consistent with previous studies [5], which confirmed that gBoost outperforms a frequent subgraph based algorithm (mine frequent subgraphs as features and then apply SVMs).

The experimental results in Fig. 4 (A) show that CogBoost outperforms igBoost. This is because the loss function in igBoost is not a cost-sensitive loss function, but heuristically adapted from the hinge loss function (i.e., simply assigning different weights to different classes). Therefore, it does not necessarily implement the Bayes decision rule and cannot guarantee minimum conditional risk.

In contrast, CogBoost- 1 and CogBoost-a adopt an optimal cost-sensitive loss function which implements the Bayes decision rule to achieve minimum cost. Evidently, both CogBoost-1 and CogBoost-a outperform gBoost over all graph datasets with significant performance gain, and outperforms igBoost for most graph datasets.

Runtime Performance The algorithm runtime in Fig. 4 (B) shows that gBoost, igBoost, and CogBoost-a all require an order of magnitude more time over CogBoost-1, Fre+CSVM, and gSemi+CSVM. For instance, CogBoost-1 only needs about 1,846 seconds on NCI-1 dataset whereas all other boosting algorithms take about over 50,000 seconds to complete the task. Overall, CogBoost-1 is 25 times faster the all other boosting algorithms. This result validates that reformulating our problem from Eq.(7) to a new problem (Eq. (10)) and using cutting plane algorithm to solve it can efficiently speed up the problem solving.

Note that Fre+CSVM and gSemi+CSVM have a little less runtime than CogBoost-1, this is because they only solve the SVM formulation (quadratic program) once, while our algorithm iteratively solves a linear program in each iterations.

Comparing the runtime of NCI and Twitter datasets, we found that although twitter dataset is significant larger than NCI, the time consumption for NCI and Twitter does not differ much. This is because the average number of nodes and edges for twitter dataset is much smaller than NCI, making it much efficient for subgraph mining for all algorithms.

Runtime Consumption Details for Boosting algorithms To better understand why CogBoost is more efficient than its peers, we investigate detailed runtime consumption in each step for boosting algorithms. These boosting methods all consist of two key steps in each iteration: i.e., optimal subgraph mining and linear problem solving. Accordingly, we report the algorithm runtime in each iteration in Fig. 6, and report average time consumption in Table 3.

Table 3 and Fig. 6 show that, on average, subgraph mining can be done in less than 20 seconds for all algorithms. At the first iteration, the subgraph mining step requires a significant amount of runtime. This is because gSpan needs to generate the search tree until the pruning condition is satisfied. Creating a new node is time consuming, because the list of embeddings is updated, and the minimality of the DFS code has to be checked (See [5] for more details). In the latter iterations, the time consumption for this process
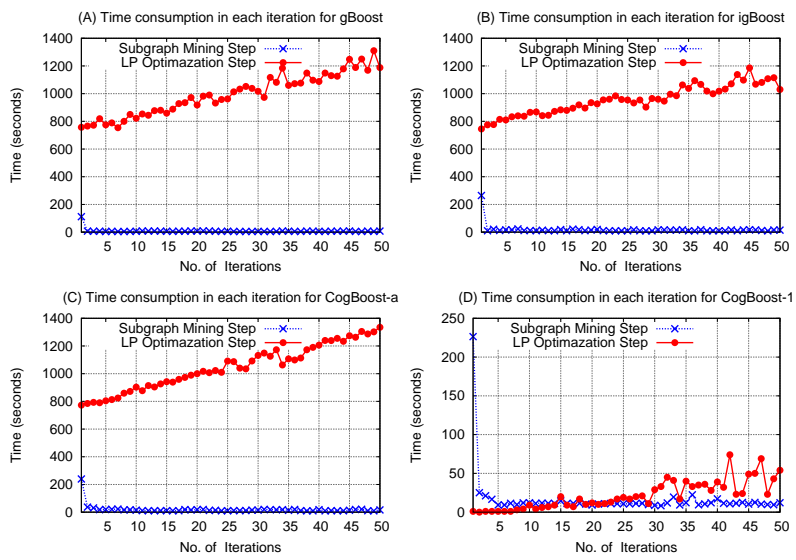

Fig. 6: Runtime performance in each iterations. Runtime consumption for (A) gBoost, (B) igBoost, (C) CogBoost-a, and (D) CogBoost-1.

TABLE 3: Average Time Consumption in Each Iteration (Seconds)

\begin{tabular}{ccccc}
\hline & gBoost & igBoost & CogBoost-a & CogBoost-1 \\
\hline Subgraph Mining & 8.24 & 18.24 & 19.39 & 16.33 \\
LP Optimazation & 993.42 & 954.66 & 1046.12 & 21.58 \\
\hline
\end{tabular}

can be reduce greatly because the searching space can be reused. The node creation is necessary only if it were not created in previous iterations. As a result, we can observe that the algorithm is more efficient in the latter iterations.

As for the LP optimization steps, gBoost, igBoost, and CogBoost-a all consume much more time than CogBoost1. This is because they all need to solve a linear problem (similar to Eq. (7) for gBoost and igBoost) with $l$ slack variables $\xi_{i \mid i=1, \cdots, l}$ in each iteration $(l$ is the total number of graph examples). When $l$ is large, it will require a very large amount of time to solve the linear problem. In contrast, CogBoost-1 solves the linear problem (Eq. (10)) with only one single slack variable $\xi$ by using cutting plane algorithms (Algorithm 3). This new formulation can greatly reduce the time required for linear problem solving.

The results in Table 3 and Fig. 6 show that CogBoost-1 only needs about 21.58 seconds for one iteration while all other algorithms require about 1,000 seconds to complete this step. Because LP optimization step is the most computationally intensive step for boosting algorithms, CogBoost1 is much faster than all existing boosting algorithms for graph classification.

Comparison of CogBoost-1 and CogBoost-a The experiment results in Fig. 4 show that CogBoost-1 can always achieve similar (or very close) classification performance as CogBoost-a. This is because CogBoost- 1 can always return an $\epsilon$-tolerance solution to CogBoost-a in each iteration. This, in fact, empirically proves the correctness of our CogBoost-1 formulation. Because CogBoost-1 can achieve accurate solutions to CogBoost-a but with much less runtime consumption than CogBoost-a, in the following experiments, we will report CogBoost-1 (termed as CogBoost) for comparison with other algorithms.

Meanwhile, we will mainly focus on the classification 

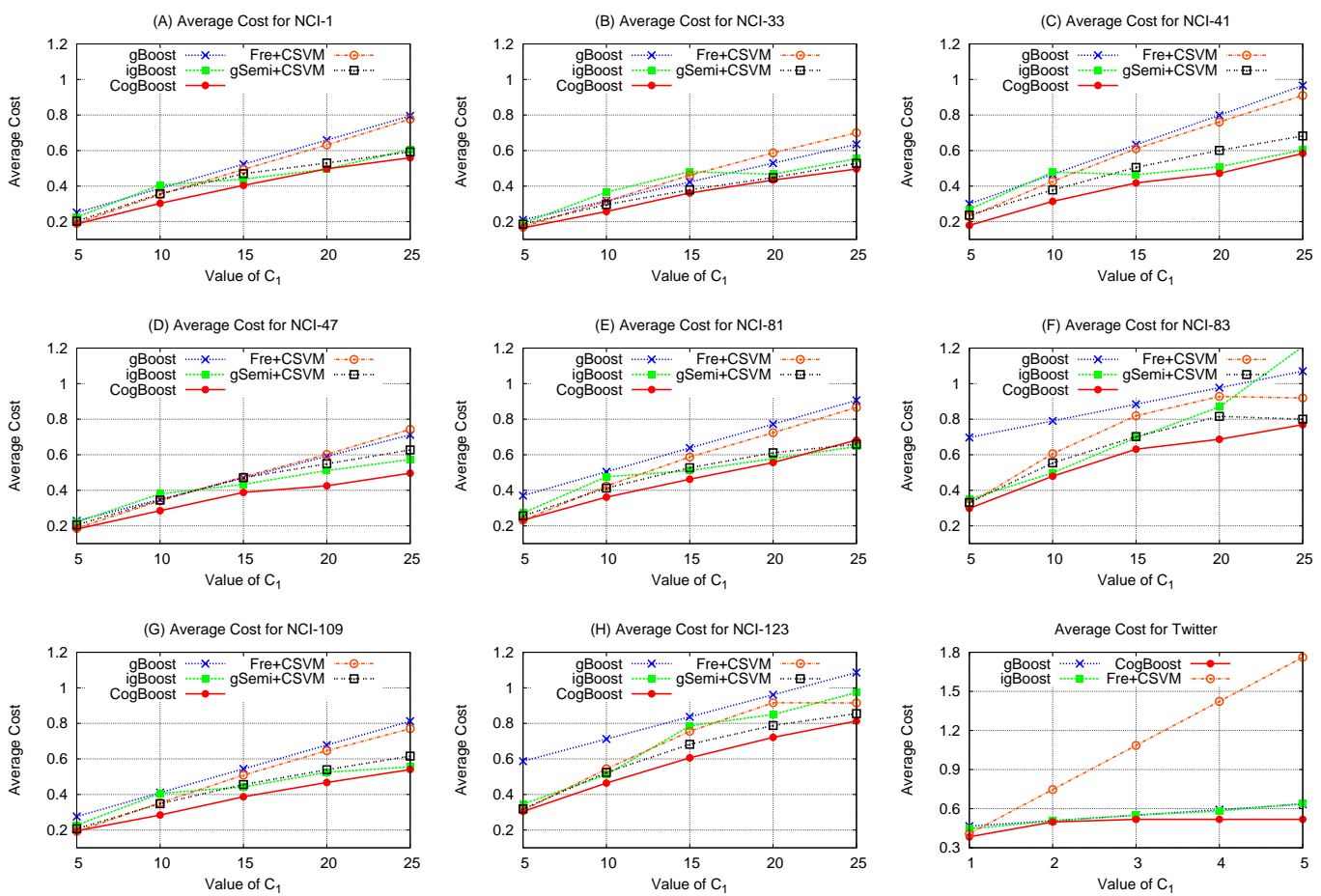

Fig. 5: Average Cost with respect to different $C_{1}$ value

performance for cost-sensitive learning because the time complexity is relatively stable for each graph dataset with the same number of training graphs.

\subsubsection{Performance w.r.t. different cost values}

In order to study the algorithm performance w.r.t. different cost values, we vary the $C_{1}$ values from 5 to 25 for NCI graphs and 1 to 5 for Twitter graphs and report the algorithm performance in Fig. 5 where the $x$-axis in each subfigure shows the $C_{1}$ values and the $y$-axis show the average costs of different methods.

Fig. 5 shows that with the increasing of $C_{1}$ value, the average costs of all all algorithms will increase. This is because the increasing of $C_{1}$ value results in a higher misclassification cost of positive graphs. Comparing to gBoost, igBoost achieves less average cost on most graph datasets. This is mainly attributed to the uneven weight assignment scheme for different classes adopted in igBoost, which allows igBoost to deal with the cost-sensitive problem to some extend.

For all datasets, CogBoost achieves minimum average cost with respect to different $C_{1}$ values. This is attributed to the optimal hinge loss function employed in CogBoost, which implements the Bayes decision rules and forces CogBoost to favor high cost samples in order to minimize the misclassification costs. This result is actually consistent with results from a previous study [22], which addresses cost-sensitive support vector machine algorithm for vector data, while CogBoost is a boosting algorithm for graph classification.
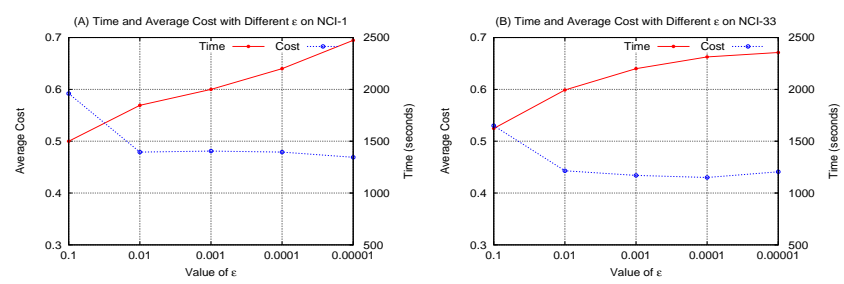

Fig. 7: Average cost (left $y$-axis) and algorithm runtime (right $y$-axis) with respect to different $\epsilon$ values ( $x$-axis). (A) NCI-1, and (B) NCI-33

\subsubsection{Performance w.r.t. different $\epsilon$ values}

In CogBoost, the parameter $\epsilon$ controls CogBoost's solutions in solving the cutting plane algorithm (Algorithm 3). In order to validate $\epsilon$ 's impact on the algorithm performance, we vary $\epsilon$ values and report CogBoost's performance in Fig. 7.

Fig. 7 shows that for large $\epsilon$ values (e.g. $\epsilon=0.1$ on NCI-1 dataset), the corresponding average cost is also large. This is because a large $\epsilon$ value returns a solution far away from the optimal solution and results in poor performance for CogBoost. As $\epsilon$ continuously decreases (from 0.1 to 0.00001 ), the average cost on both NCI-1 and NCI-33 datasets decrease. This is because with a small $\epsilon$ value, CogBoost can return accurate solution for classification. However, the runtime consumption for smaller $\epsilon$ values will also increase because more iterations are required in the cutting algorithm. Our empirical results suggest that a moderate value (such as $\epsilon=0.01$ ) has a good tradeoff between time complexity and average cost. So we set $\epsilon=0.01$ as a default value in our experiments. 
In summary, our experiments suggest that cost-sensitive graph classification is a much more complicated problem than traditional cost-sensitive learning, mainly because that graph classification heavily replies on subgraph feature exploration. Simply converting a graph dataset into a vector representation, by using frequent subgraph features, and then applying cost-sensitive learning (like Fre+CSVM does) is far from optimal. Indeed, subgraph features play vital role for graph classification. By using a cost-sensitive subgraph exploration process and a cost-sensitive loss function, CogBoost demonstrates its superb performance for cost-sensitive graph classification.

\section{Conclusion}

In this paper, we formulated a cost-sensitive graph classification problem for large scale graph datasets. We argued that many real-world applications involve data with dependency structures and the cost of misclassifying samples in different classes is inherently different. This problem motivates us to consider effective graph classification algorithms with cost-sensitive capability and being suitable for large scale graph datasets. To solve the problem, we proposed a fast boosting algorithm, CogBoost, which embeds the costs into the subgraph exploration and the learning process. The boosting procedure utilizes an optimal loss function to minimize the misclassification costs by implementing the Bayes decision rule. To enable fast training on large scale graphs, a cutting plane formulation is derived so that the linear problem can be solved efficiently in each iteration. Experimental results on large real-life graph datasets validate our designs.

\section{ACKNOWLEDGMENT}

We thank the constructive comments from anonymous reviewers. This work is partially supported by Australia ARC Discovery Project (DP140102206), and National Scholarship for Building High Level Universities, China Scholarship Council (No. 2011630030).

\section{REFERENCES}

[1] H. Kashima, K. Tsuda, and A. Inokuchi, Kernels for Graphs. Cambridge (Massachusetts): MIT Press, 2004, ch. In: Schlkopf B, Tsuda K, Vert JP, editors. Kernel methods in computational biology.

[2] M. Fang and D. Tao, "Networked bandits with disjoint linear payoffs," in Proc. 20th ACM SIGKDD. ACM, 2014, pp. 11061115.

[3] S. Pan, X. Zhu, C. Zhang, and P. S. Yu, "Graph stream classification using labeled and unlabeled graphs," in Proc. of ICDE. IEEE, 2013.

[4] K. Riesen and H. Bunke, "Graph classification by means of lipschitz embedding," IEEE Trans. on SMC - B, vol. 39, pp. 1472-1483, 2009.

[5] H. Saigo, S. Nowozin, T. Kadowaki, T. Kudo, and K. Tsuda, "gboost: a mathematical programming approach to graph classification and regression," Machine Learning, vol. 75, pp. 69-89, 2009.

[6] Y. Zhu, J. Yu, H. Cheng, and L. Qin, "Graph classification: a diversified discriminative feature selection approach," in Proc. of CIKM. ACM, 2012, pp. 205-214.

[7] X. Kong and P. Yu, "Semi-supervised feature selection for graph classification," in Proc. of ACM SIGKDD, 2010.

[8] J. Tang and H. Liu, "An unsupervised feature selection framework for social media data," IEEE Transactions on Knowledge and Data Engineering, vol. 26, no. 12, pp. 2914-2927, 2014.
[9] H. Fei and J. Huan, "Boosting with structure information in the functional space: an application to graph classification," in Proc. of $A C M$ SIGKDD, 2010.

[10] J. Wu, X. Zhu, C. Zhang, and P. Yu, "Bag constrained structure pattern mining for multi-graph classification," Knowledge and Data Engineering, IEEE Transactions on, vol. PP, no. 99, pp. 1-1, 2014.

[11] S. Pan and X. Zhu, "Graph classification with imbalanced class distributions and noise," in IJCAI, 2013.

[12] P. Tiwari, J. Kurhanewicz, and A. Madabhushi, "Multi-kernel graph embedding for detection, gleason grading of prostate cancer via mri/mrs," Medical Image Analysis, vol. 17, no. 2, pp. 219-235, 2013.

[13] M. Deshpande, M. Kuramochi, N. Wale, and G. Karypis, "Frequent substructure-based approaches for classifying chemical compounds," IEEE TKDE, vol. 17, pp. 1036-1050, 2005.

[14] P. Domingos, "Metacost: a general method for making classifiers cost-sensitive," in Proc. of ACM KDD, 1999, pp. 155-164.

[15] K. Veropoulos, C. Campbell, and N. Cristianini, "Controlling the sensitivity of support vector machines," in Proc. of IJCAI, vol. 1999, 1999, pp. 55-60.

[16] C. Elkan, "The foundations of cost-sensitive learning," in International joint conference on artificial intelligence, vol. 17, no. 1. Citeseer, 2001, pp. 973-978.

[17] S. Zhang, Z. Qin, C. X. Ling, and S. Sheng, "Missing is useful": missing values in cost-sensitive decision trees," IEEE TKDE, vol. 17, no. 12, pp. 1689-1693, 2005.

[18] F. R. Bach, D. Heckerman, and E. Horvitz, "Considering cost asymmetry in learning classifiers," The Journal of Machine Learning Research, vol. 7, pp. 1713-1741, 2006.

[19] B. Zadrozny, J. Langford, and N. Abe, "Cost-sensitive learning by cost-proportionate example weighting," in Proc. of IEEE ICDM, 2003.

[20] H. Masnadi-Shirazi and N. Vasconcelos, "Cost-sensitive boosting," IEEE PAMI, vol. 33, no. 2, pp. 294-309, 2011.

[21] A. C. Lozano and N. Abe, "Multi-class cost-sensitive boosting with p-norm loss functions," in Proc. of ACM KDD, 2008, pp. 506-514.

[22] H. Masnadi-Shirazi and N. Vasconcelos, "Risk minimization, probability elicitation, and cost-sensitive svms," in ICML, 2010.

[23] N. Abe, B. Zadrozny, and J. Langford, "An iterative method for multi-class cost-sensitive learning," in Proc. ACM KDD, 2004, pp. $3-11$.

[24] X. Zhu and X. Wu, "Class noise handling for effective costsensitive learning by cost-guided iterative classification filtering," IEEE TKDE, vol. 18, no. 10, pp. 1435-1440, 2006.

[25] S. Lomax and S. Vadera, "A survey of cost-sensitive decision tree induction algorithms," ACM Computing Surveys, vol. 45, no. 2, 2013.

[26] G. K. J. Shawe-Taylor, "Optimizing classifiers for imbalanced training sets," in NIPS, vol. 11. MIT Press, 1999, p. 253.

[27] X. Yan and J. Han, "gspan: Graph-based substructure pattern mining," in Proc. of ICDM, Maebashi City, Japan, 2002.

[28] X. Wu, X. Zhu, G. Wu, and W. Ding, "Data mining with big data," IEEE TKDE, vol. 26, no. 1, pp. 97-107, 2014.

[29] S. Ranu and A. Singh, "Graphsig: A scalable approach to mining significant subgraphs in large graph databases," in Proc. of ICDE. IEEE, 2009, pp. 844-855.

[30] J. Wu, Z. Hong, S. Pan, X. Zhu, C. Zhang, and Z. Cai, "Multi-graph learning with positive and unlabeled bags," in SIAM International Conference on Data Mining. SIAM, 2014, pp. 1-12.

[31] H. Fei and J. Huan, "Structure feature selection for graph classification," in Proc. of ACM CIKM, California, USA, 2008.

[32] N. Jin, C. Young, and W. Wang, "Graph classification based on pattern co-occurrence," in Proc. of ACM CIKM, 2009.

[33] M. Thoma, H. Cheng, A. Gretton, J. Han, H. Kriegel, A. Smola, L. Song, P. Yu, X. Yan, and K. Borgwardt, "Near-optimal supervised feature selection among frequent subgraphs," in Proc. of SDM, 2009.

[34] T. Kudo, E. Maeda, and Y. Matsumoto, "An application of boosting to graph classification," in Proc. of NIPS, Vancouver, Canada, 2004.

[35] J. Wu, S. Pan, X. Zhu, and Z. Cai, "Boosting for multi-graph classification," IEEE transactions on cybernetics, 2014.

[36] S. Pan, J. Wu, X. Zhu, and C. Zhang, "Graph ensemble boosting for imbalanced noisy graph stream classification." IEEE transactions on cybernetics, 2014.

[37] W. Fan, S. J. Stolfo, J. Zhang, and P. K. Chan, "Adacost: misclassification cost-sensitive boosting," in ICML, 1999, pp. 97-105.

[38] H. Masnadi-Shirazi, N. Vasconcelos, and A. Iranmehr, "Costsensitive support vector machines," arXiv:1212.0975, 2012. 
[39] R. Tibshirani, "Regression shrinkage and selection via the lasso," Journal of the Royal Statistical Society. Series B (Methodological), pp. 267-288, 1996.

[40] S. Nash and A. Sofer, "Linear and nonlinear programming," Newyark McGraw-Hill, 1996.

[41] T. Joachims, "Training linear svms in linear time," in $K D D, 2006$, pp. 217-226.

[42] N. Megiddo, "On the complexity of linear programming," Advances in economic theory, pp. 225-268, 1987.

\section{Appendix A}

\section{A.1 Duality of Eq. (7)}

The Lagrangian function of Eq.(7) can be written as:

$$
\begin{aligned}
L \quad & (\boldsymbol{\xi}, \boldsymbol{w}) \\
& =\|\boldsymbol{w}\|+\frac{C}{l}\left\{C_{1} \sum_{\left\{i \mid y_{i}=1\right\}} \xi_{i}+\gamma \sum_{\left\{j \mid y_{j}=-1\right\}} \xi_{j}\right\} \\
& +\sum_{i \mid y_{i}=1} \mu_{i}\left\{1-\xi_{i}-\sum_{k=1}^{m} w_{k} \cdot \hbar_{g_{k}}\left(G_{i}\right)\right\} \\
& +\sum_{j \mid y_{j}=-1} \mu_{j}\left\{\sum_{k=1}^{m} w_{k} \cdot \hbar_{g_{k}}\left(G_{j}\right)+\frac{1}{\gamma}-\xi_{j}\right\} \\
& -\sum_{i=1}^{m} q_{k} \cdot w_{k}-\sum_{i=1}^{l-} p_{i} \cdot \xi_{i}-\sum_{j=1}^{l+} p_{j} \cdot \xi_{j}
\end{aligned}
$$

Where, we have $\mu_{i} \geq 0, \mu_{j} \geq 0, p_{i} \geq 0, q_{k} \geq 0$.

At optimum, the first derivative of the Lagrangian w.r.t. the primal variables $(\boldsymbol{\xi}, \boldsymbol{w})$ must vanish,

$$
\begin{aligned}
\frac{\partial L}{\partial \xi_{i \mid y_{i}=1}} & =\frac{C C_{1}}{l}-\mu_{i}-p_{i}=0 \Rightarrow 0 \leq \mu_{i} \leq \frac{C C_{1}}{l} \\
\frac{\partial L}{\partial \xi_{j \mid y_{j}=-1}} & =\frac{C \gamma}{l}-\mu_{j}-p_{j}=0 \Rightarrow 0 \leq \mu_{j} \leq \frac{C \gamma}{l} \\
\frac{\partial L}{\partial w_{k}} & \Rightarrow 1-\sum_{i} \mu_{i} \hbar_{g_{k}}\left(G_{i}\right)+\sum_{j} \mu_{j} \hbar_{g_{k}}\left(G_{j}\right)-q_{k}=0 \\
& \Rightarrow \sum_{i} \mu_{i} \hbar_{g_{k}}\left(G_{i}\right)-\sum_{j} \mu_{j} \hbar_{g_{k}}\left(G_{j}\right)<1
\end{aligned}
$$

Note that $\frac{\partial L}{\partial w_{k}}$ with respect to $w_{k}$ equals to 1 because $w_{k} \geq$ 0 . Substituting these variables in Eq. (14), we obtain its dual problem as Eq. (8).

\section{A.2 Equality of Eq. (7) and Eq. (10)}

Here we will prove that given any solution $\boldsymbol{w}$ of Eq. (10), it will be also the solution of Eq. (7).

Given a $\boldsymbol{w}$, the $\xi_{i}$ and $\xi_{j}$ in Eq. (7) can be optimized independently, i.e., $\xi_{i}=\max \left(0,1-\boldsymbol{w}^{T} \boldsymbol{x}_{i}\right)$ and $\xi_{j}=$ $\max \left(0,1 / \gamma+\boldsymbol{w}^{T} \boldsymbol{x}_{j}\right)$. For Eq. (10), the optimal $\xi$ for a given $\boldsymbol{w}$ is:

$$
\begin{aligned}
& \max _{c \in\{0,1\}^{l}} \frac{1}{l}\left\{C_{1} \sum_{y_{i}=1} c_{i}+\sum_{y_{j}=1} c_{j}\right\} \\
& -\frac{1}{l} \boldsymbol{w}^{T}\left\{C_{1} \sum_{y_{i}=1} c_{i} \boldsymbol{x}_{i}-\gamma \sum_{y_{j}=-1} c_{j} \boldsymbol{x}_{j}\right\} \\
& =\quad 1 / l \sum_{y_{i}=1} \max _{\boldsymbol{c} \in\{0,1\}^{l}}\left(C_{1} c_{i}-C_{1} c_{i} \boldsymbol{w}^{T} \boldsymbol{x}_{i}\right) \\
& +1 / l \sum_{y_{j}=1} \max _{\boldsymbol{c} \in\{0,1\}^{l}}\left(c_{j}+c_{j} \gamma \boldsymbol{w}^{T} \boldsymbol{x}_{j}\right) \\
& =\quad C_{1} / l \sum_{y_{i}=1}^{y_{j}} \max \left(0,1-\boldsymbol{w}^{T} \boldsymbol{x}_{i}\right) \\
& +\gamma / l \sum_{y_{j}=1}^{y_{i}=1} \max \left(0,1 / \gamma+\boldsymbol{w}^{T} \boldsymbol{x}_{j}\right) \\
& =\left\{C_{1} \sum_{\left\{i \mid y_{i}=1\right\}}^{y_{j}=1} \xi_{i}+\gamma \sum_{\left\{j \mid y_{j}=-1\right\}} \xi_{j}\right\} / l=\xi
\end{aligned}
$$

Therefore, the objective functions of Eq. (7) and Eq. (10) are equal for any $\boldsymbol{w}$ given the optimal $\boldsymbol{\xi}$ and $\xi$, i.e., they are equivalent.

\section{A.3 Duality of Eq.(10)}

Here we derive the duality of Eq.(10). The Lagrangian function of Eq.(10) can be written as:

$$
\begin{aligned}
L(\xi, \boldsymbol{w}) & =\|\boldsymbol{w}\|+C \xi \\
& -\sum_{\boldsymbol{c}} \lambda_{\boldsymbol{c}}\left\{\frac{1}{l} \boldsymbol{w}^{T}\left(C_{1} \sum_{y_{i}=1} c_{i} \boldsymbol{x}_{i}-\gamma \sum_{y_{j}=-1} c_{j} \boldsymbol{x}_{j}\right)\right. \\
& \left.-\frac{1}{l}\left(C_{1} \sum_{y_{i}=1} c_{i}+\sum_{y_{j}=1} c_{j}\right)+\xi\right\}-\sum_{i=1}^{m} q_{k} \cdot w_{k}-p \xi
\end{aligned}
$$

Where, we have $\lambda_{c} \geq 0, p \geq 0, q_{k} \geq 0$.

Similarly, we take the first derivative of the Lagrangian w.r.t. the primal variables $(\xi, \boldsymbol{w})$,

$$
\begin{aligned}
\frac{\partial L}{\partial \xi} & =C+\sum_{\boldsymbol{c}} \lambda_{c}-p=0 \Rightarrow 0 \leq \sum_{\boldsymbol{c}} \lambda_{c} \leq C \\
\frac{\partial L}{\partial w_{k}} & \Rightarrow 1-\frac{C_{1}}{l} \sum_{\boldsymbol{c}} \lambda_{\boldsymbol{c}} \sum_{i} c_{i} \boldsymbol{x}_{i}^{k}+\frac{\gamma}{l} \sum_{\boldsymbol{c}} \lambda_{\boldsymbol{c}} \sum_{j} c_{j} \boldsymbol{x}_{j}^{k}-q_{k}=0 \\
& \Rightarrow \frac{C_{1}}{l} \sum_{\boldsymbol{c}} \lambda_{\boldsymbol{c}} \sum_{i} c_{i} \boldsymbol{x}_{i}^{k}-\frac{\gamma}{l} \sum_{\boldsymbol{c}} \lambda_{\boldsymbol{c}} \sum_{i} c_{j} \boldsymbol{x}_{j}^{k}<1
\end{aligned}
$$

Substituting these variables in Eq. (15), we obtain the its dual problem as Eq. (11).

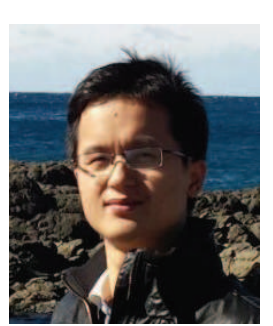

Shirui Pan received his master degree in computer science from Northwest A\&F University, Yangling, Shaanxi, China, in 2011. Since September 2011, he has been working toward the PhD degree in the Centre for Quantum Computation and Intelligent Systems (QCIS), Faculty of Engineering and Information Technology, University of Technology, Sydney (UTS). His research focuses on data mining and machine learning.

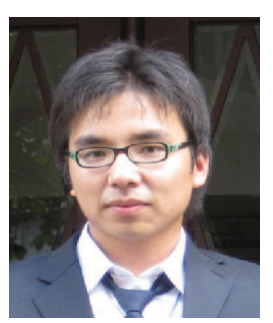

Jia Wu received his bachelor degree in computer science from China University of Geosciences (CUG), Wuhan, China, in 2009. Since September 2009, he has been working toward the $\mathrm{PhD}$ degree under the MasterDoctor combined program in computer science from CUG. Besides, he is also pursuing a PhD degree in QCIS Centre, Faculty of Engineering and Information Technology, University of Technology, Sydney (UTS), Ausand machine learning. tralia. His research focuses on data mining

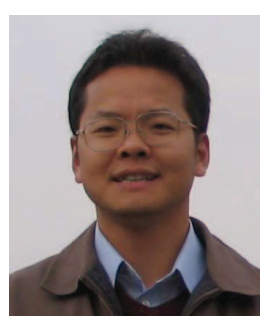

Xingquan Zhu received the $\mathrm{PhD}$ degree in computer science from Fudan University, Shanghai, China. He is an associate professor in the Department of Computer \& Electrical Engineering and Computer Science, Florida Atlantic University. Prior to that, he was with the Centre for Quantum Computation \& Intelligent Systems, University of Technology, Sydney, Australia. His research interests mainly include data mining, machine learning, and multimedia systems. Since 2000, he has published more than 180 refereed journal and conference papers in these areas, including two Best Paper Awards and one Best Student Paper Award. 